\title{
Badiou en Argentina. Acontecimiento y las vicisitudes de una recepción temprana y política
}

\author{
Marcelo Starcenbaum \\ Universidad Nacional de La Plata - Consejo Nacional de Investigaciones Científicas y Técnicas \\ mstarcenbaum@gmail.com
}

Title: Badiou in Argentina. Acontecimiento: An Early and Political Reception

Resumen: El objetivo de este trabajo es demostrar que la recepción de Alain Badiou en Argentina tuvo un carácter temprano y político. Para ello, analizaremos la revista Acontecimiento, que durante veinticinco años llevó a cabo una lectura de la realidad argentina y latinoamericana a través de tesis y conceptos provenientes de la obra del filósofo francés. En la primera mitad de la década de 1990 la revista procesará el derrumbe del socialismo real y la imposición de una gramática política democrática. En la segunda mitad de la década de 1990, diferentes experiencias políticas serán caracterizadas como una nueva secuencia política desplegada a distancia de los partidos y el Estado.

Palabras clave: Badiou - Argentina - marxismo - recepción

\begin{abstract}
The aim of this paper is to demonstrate that the reception of Alain $\mathrm{Ba}-$ diou in Argentina had an early and political character. We will analyze the journal Acontecimiento, which for twenty-five years led to a reading of the Argentine and Latin American reality through theses and concepts from the work of the French philosopher. In the first half of the 1990s, the journal will process the collapse of real socialism and the imposition of a democratic political grammar. In the second half of the 1990s, different political experiences will be characterized as a new political sequence deployed at a distance from the parties and the State. Keywords: Badiou - Argentine - Marxism - Reception
\end{abstract}

Recepción: 25 de noviembre de 2020. Aceptación: 15 de febrero de 2021 
I. En un artículo de 2001 publicado en inglés en una revista especializada de filosofia, Bruno Bosteels consideró necesario agregar que mientras sólo recientemente había obtenido la atención del mundo angloparlante, la obra de Badiou siempre había desempeñado un rol activo y militante en América Latina. ${ }^{1}$ Mencionaba asismismo a una revista argentina que por más de una década había publicado traducciones de Badiou y había producido una serie de intervenciones sobre experiencias políticas como las de las Madres de Plaza de Mayo y las de los zapatistas. El hecho de que los países latinoamericanos y la militancia política irrumpieran en una publicación de tales características se nos presenta como sintomático de las particularidades de la circulación internacional de la obra de Badiou. Bosteels, quien fue una figura clave en la colocación de Badiou en el mundo angloparlante (e incluso en el francés), y a su vez un conocedor (y en algún sentido protagonista) de la difusión del filósofo francés en América Latina, le informaba al lector de su artículo no sólo de la existencia de una importante recepción de Badiou en los países latinoamericanos sino también que dicha recepción había sido anterior a la del ámbito de habla inglesa y, a diferencia de ésta, se había producido en vinculación con la militancia política. El otro elemento significativo del pasaje citado es la mención a una revista argentina que había difundido la obra de Badiou en Argentina y que había utilizado dicho trabajo para discutir determinados procesos políticos desarrollados en la región. El objetivo de este trabajo es dar un paso más allá de la constatación de la heterogeneidad en la circulación internacional de la obra de Badiou y de la existencia de una experiencia de recepción temprana y política en América Latina. Tomando como punto de partida estas dos variables, que conforman lo que Bosteels (2011, p. XIII) denominará posteriormente "la hipótesis histórica y geo-

1. La obra de Alain Badiou (1937) constituye una de las intervenciones más potentes y singulares del marxismo contemporáneo. El trabajo del filósofo francés comenzó en la década de 1960 bajo el influjo de la renovación del marxismo emprendida por su maestro Louis Althusser. De filiación política maoísta, formó parte del proceso de radicalización desplegado en los círculos intelectuales y políticos franceses entre fines de la década de 1960 y principios de 1970. En la década de 1980, desarrolló una productiva -aunque como veremos, solitaria- aproximación a Lacan. Con la crisis definitiva del socialismo real se ubicó a distancia tanto de aquellos que negaban - o evitaban a toda costa- la debacle de la experiencia comunista como de aquellos que la celebraban en nombre del advenimiento de la democracia capitalista. En los últimos treinta años se dedicó a la construcción de un sistema filosófico propio. A su vez, desplegó en Francia una fuerte militancia política, la cual se expresó tanto en la conformación de agrupamientos intelectuales como en el apoyo a diversas causas sociales. Como se verá a continuación, las interpretaciones en torno a este itinerario político-intelectual -así como las referencias a través de las cuales éste puede ser reconstruido- forman parte de la propia reconstrucción que aquí proponemos. 
gráfica" en el estudio de la obra de Badiou, nos concentraremos en la revista Acontecimiento como una experiencia de recepción de la obra del filósofo francés en Argentina. Colocando esta experiencia en el marco general de los desfases y diferencias entre los itinerarios de Badiou en el mundo angloparlante y en el contexto latinoamericano, analizaremos las lecturas de Badiou realizadas en el seno de Acontecimiento y los modos en los que estas lecturas se vincularon con los procesos políticos desarrollados en Argentina y en la región.

La inscripción de Acontecimiento en el proceso más amplio de la circulación internacional de la obra de Badiou resulta indispensable para alcanzar una caracterización de las particularidades de su recepción en Argentina. Es decir, la afirmación de que la recepción argentina de Badiou constituyó un fenómeno temprano y político sólo es posible a partir de la constatación de las particularidades de su recepción en el mundo de habla inglesa. Con respecto al primer rasgo, existe consenso en que las primeras lecturas sistemáticas de Badiou en ese ámbito se produjeron a fines de la década de 1990 a través de los trabajos de Hallward (1998) y Žižek (1999). Poco tiempo después, al publicar su aproximación sistemática, Hallward (2003, p. 349) constatará con sorpresa que Badiou ni siquiera era mencionado en las investigaciones sobre filosofia francesa producidas en Inglaterra. En el mismo sentido, Feltham (2010, p. 9) destacará la particularidad de Badiou en tanto filósofo que obtuvo un reconocimiento tardío en el mundo angloparlante. En Argentina, y por extensión en América Latina, Badiou ha sido una referencia familiar desde fines de la década de 1960. En 1969, su texto "El (re)comienzo del materialismo dialéctico" fue incluido por José Aricó en el Cuaderno de Pasado y Presente Materialismo histórico y materialismo dialéctico. En 1974 Ricardo Piglia publicó su texto "La autonomía del proceso estético" en una compilación titulada Literatura y sociedad. Como veremos a continuación, Cerdeiras comenzó su trabajo de lectura y difusión a comienzos de la década de 1980, momento en el que, como destacan Ashton, Bartlett y Clemens (2006, p. 8) la obra de Badiou se producía en absoluta soledad y con una repercusión casi nula en el debate filosófico europeo.

Con respecto al segundo rasgo de la recepción argentina se impone una aclaración. La referencia a una recepción política no descansa sobre una concepción ingenua según la cual podría haber lecturas no politicas. Se refiere más bien a una característica que surge de inmediato al contrastar su recepción con la del ámbito de habla inglesa. En este punto también existe consenso en cuanto a que la recepción en el mundo angloparlante tuvo lugar especialmente en el ámbito académico. Según Žižek (2003, p. xi), la obra de Badiou resultó potente y productiva entre los intelectuales de habla inglesa en tanto habilitaba una crítica a los 
fundamentos de la democracia liberal, las políticas de la identidad del multiculturalismo y la lectura deconstruccionista del marxismo. Por otra parte, Bosteels (2011) ha advertido dos operaciones producidas en el mundo angloparlante en relación a la trayectoria militante del propio Badiou: la desatención casi unánime -a excepción de Hallward (2003)de la historia de las organizaciones políticas integradas o dirigidas por Badiou como la Union des Communistes de France marxiste-léniniste (UCFML) y la Organisation Politique (OP) -así como sus respectivas publicaciones Le Perroquet y La Distance Politique- y el consecuente borramiento de la problemática maoísta en su obra. Por último, la preponderancia de una recepción académica relegó los ejercicios de uso o aplicación de la obra de Badiou para el análisis de problemas politicos concretos. Al respecto, Bartlett y Clemens (2010, p. 4) han señalado la rareza del artículo de Feltham (2004) en el que los conceptos del filósofo francés son utilizados para pensar una experiencia política. En efecto, el trabajo de Feltham constituyó un ejercicio excepcional en el que la instalación de una carpa aborigen frente al Parlamento australiano era entendida en términos de un acontecimiento en tanto la afirmación de los derechos de las poblaciones originarias sobre la tierra constituía una disrupción que inauguraba un proceso de cambio. La experiencia de Acontecimiento demuestra que la recepción argentina de Badiou, además de temprana, fue preponderantemente política. Por un lado, porque no se produjo en espacios académicos sino en el campo de la izquierda intelectual y vinculado a procesos políticos concretos. Por el otro, porque la militancia política de Badiou y su vínculo con el maoísmo fueron elementos presentes en la apropiación realizada por la revista de la obra del filósofo francés. Finalmente, porque si bien la revista acogió reflexiones de índole teórica, produjo análisis significativos de experiencias politicas desarrolladas en el país y en la región. ${ }^{2}$

Resulta atinado el señalamiento metodológico de que, en tanto estructura de sociabilidad, las revistas no se encarnan tanto en un grupo de personas sino más bien en una de ellas, que es la que le da el impulso principal a la publicación (Beigel, 2003; Pluet-Despatin, 1992). En el caso de Acontecimiento, este rol le correspondió indudablemente a Raúl Cerdeiras. Cerdeiras comenzó su militancia política a fines de la

2. No tenemos el espacio suficiente para explayarnos acerca de la dimensión editorial de esta recepción temprana. Constatemos al menos que los trabajos más importantes de Badiou fueron traducidos al castellano muchos años antes que la traducción al inglés: El concepto de modelo, de 1969, fue publicado en castellano en 1976 y en inglés en 2007; Teoría del sujeto, de 1982, en castellano en 2008 y en inglés en 2013; El ser y el acontecimiento, de 1988, en castellano en 1999 y en inglés en 2005; La ética, de 1993, en castellano en 1994 y en inglés en 2001. Si bien desactualizada, puede verse una bibliografia de la obra de Badiou en inglés en Ashton (2006). 
década de 1950 en el grupo de Silvio Frondizi. Graduado en derecho, se vinculó tempranamente a los grupos de estudio que florecieron durante el onganiato y que estuvieron fuertemente marcados por la crítica al marxismo-leninismo de matriz soviética. Participó en el grupo de Raúl Sciarreta, un filósofo que venía de romper con el Partido Comunista argentino y que fue uno de los difusores de Althusser y Lacan en Argentina. En los comienzos de la década de 1970 estuvo vinculado con los sectores de la izquierda peronista y participó en las "Cátedras Nacionales" de la Universidad de Buenos Aires (UBA). Durante la dictadura estuvo abocado a la lectura de Althusser, Lacan y Heidegger. Con el regreso de la democracia toma contacto con Théorie du sujet, libro publicado por Badiou en 1982. Comienza un intercambio epistolar con Badiou y en 1986 viaja a Francia. Allí participa de sus clases en la Universidad, se interioriza en su trabajo y su militancia, y accede a borradores de $L$ 'Être et l'Événement. En aquellos años coordina grupos de estudio en Buenos Aires en los que introduce la obra de Badiou. Así como las revistas suelen encarnarse en una persona, también constituyen espacios de encuentro de itinerarios intelectuales que comparten un credo común o que son iniciados en él por el individuo aglutinante (Pluet-Despatin, 1992). Es así que, junto a Cerdeiras, participaron en la creación de la revista un grupo de personas que habían formado parte de sus grupos de estudio y que estaban fuertemente marcadas por la obra de Badiou. Por un lado, Alejandro Cerletti, graduado en filosofia y docente de la UBA especializado en filosofia de la educación y enseñanza de la filosofia. Por otro lado, Ignacio Lewkowicz, un graduado de historia que venía de romper con el PCA. Finalmente, Diego Zerba, graduado de psicología y también docente de la UBA. Zerba había formado parte junto a Cerdeiras del grupo de estudio de Sciarreta y había participado en la formación del Centro Educativo de Devoto (CUD) -una experiencia de autogestión educativa realizada por los presos de Devoto en articulación con la UBA-. También había militado en el Movimiento al Socialismo (MAS), en cuyo Congreso de 1990 había presentado un texto fuertemente influenciado por las tesis de Badiou -luego publicado en el número 3 de la revista con el título "Para un replanteo de la política"-. Como dice Beatriz Sarlo (1992), toda experiencia de publicación periódica comienza cuando alguien pronuncia la frase "publiquemos una revista". Es así como, a instancias de Cerdeiras, en julio de 1991 sale el primer número de Acontecimiento. Revista para pensar la politica. ${ }^{3}$

3. La composición del Comité Editorial de la revista fue variando a través de los años. Hasta el número 3 (1992) contó únicamente con Cerdeiras como director y editor responsable. Desde el número 4 (1992) hasta el 6 (1993) funcionó un Comité Editorial compuesto por Valeria Hall, Dicky Miur, Cerletti, Lewkowciz y Zerba. En el 


\section{$* * *$}

II. Como también ha sido señalado, los manifiestos inaugurales constituyen textos importantes para dar cuenta de la personalidad de una revista, la construcción de su proyecto y el espacio que aspira a ocupar en el campo intelectual de una época (Beigel, 2003; Pita y Grillo, 2013; Pluet-Despatin, 1992). La revista Acontecimiento no representa una excepción a la riqueza heurística que suelen presentar este tipo de materiales. El "Manifiesto político" de Cerdeiras presentó de manera sistemática la voluntad de intervención de Acontecimiento en los debates de la izquierda intelectual argentina. ${ }^{4}$ El texto que abrió el primer número de la revista, y que actuó como soporte de su intervención a lo largo de toda su experiencia, estaba dominado por las conclusiones que Badiou había desarrollado en la segunda mitad de la década de 1980 -aquellas plasmadas en los textos Peut-on penser la politique? y Manifeste pour la philosophie-. A través de una serie de paráfrasis de dicho trabajo, Acontecimiento proponía una intervención centrada en la crítica a las formas predominantes de la política (centralidad del Estado y los partidos, vaciamiento de la inventiva, primado del discurso jurídico y económico), el diagnóstico de la situación del marxismo (agotamiento de su productividad histórica, necesidad de asumir su muerte, esterilidad de su defensa), y la propuesta de un nuevo modo de pensar y hacer la política (autonomía de otras prácticas sociales, desligamiento del lazo social, separación del Estado, no representación). Sobre la inscripción de Acontecimiento en un modo ya tradicional de irrumpir en el espacio público, dos elementos metadiscursivos del manifiesto de Cerdeiras nos permiten una mayor comprensión de la singularidad de la intervención de la revista. A diferencia de la abundancia textual que suele primar en los manifiestos, en este caso se trataba de un despliegue de afirmaciones concisas enunciadas a modo de tesis y numeradas de manera consecutiva. Y de manera contrapuesta a los procesos de rectificación

número 7 (1994) el Comité quedó conformado por Lucía Scrimni, Inés Lequio -con intermitencias- y Cerletti. A partir del número 23 (2002) pasaron a formar parte del Comité Gabriel D’Iorio, Elsa González, Pablo Kohen, Alberto Migones, Blas Matías Michienzi, Andrés Pezzola, Víctor Militello, Lequio y Cerletti -sumándose Bosteels en el número 29-30 (2005). En la última etapa de la revista también participaron como miembros del Comité Roxana Yattah, Marina Di Carlo, Martín López, Hernán Mancuso y Juan Cerdeiras. En sus primeros años, la revista mantuvo vínculos con el colectivo Malgré Tout, liderado en Francia por el filósofo argentino exiliado Miguel Benasayag. A su vez, la revista tuvo una expresión política y organizativa a través del Grupo Acontecimiento, también liderado por Cerdeiras e integrado por los sucesivos miembros del Comité Editorial.

4. Raúl Cerdeiras, "Manifiesto político", en Acontecimiento, n 1, 1991, pp. 10-18. 
a los que suelen ser sometidos los manifiestos en etapas subsiguientes de la publicación, en este caso una versión resumida de la tesis fue mantenida durante los veinticinco años de la revista en una sección titulada "Dijimos en el primer número de Acontecimiento".

Sin que se modificara el marco general de la intervención de la revista, de lo cual da cuenta la vigencia del manifiesto durante los cuarenta y ocho números, la experiencia de Acontecimiento estuvo atravesada por una serie de modulaciones, especialmente en lo relativo a los procesos históricos privilegiados y los sujetos políticos jerarquizados. Estos énfasis y desplazamientos, previsibles en gran medida en una intervención intelectual sostenida durante veinticinco años, pueden ser captados a partir de las diferentes secciones de la revista. En primer lugar, los artículos del propio Cerdeiras, los cuales operaron a modo de vertebradores de cada uno de los números. En los primeros años de la década de 1990, dichos textos estuvieron centrados en el derrumbe de la experiencia socialista y los efectos que este proceso tenía para la teoría marxista y la política emancipatoria. Uno de ellos, reproducido también en el primer número, da cuenta de la productividad de la tesis de Badiou acerca de la necesidad de la destrucción del marxismo. La idea de que en el Estado socialista, en la clase obrera y en los movimientos de liberación se cifraba la capacidad del marxismo para estructurar la historia, pero también su incapacidad contemporánea para politizar la vida de los pueblos, habilitaba una dislocación de los términos predominantes en los cuales se analizaba la relación entre el fracaso del socialismo real y la crisis del marxismo. Por este camino se señalaba el tenor reaccionario de las posiciones que abordaban dicha relación a partir de las categorías de triunfo y fracaso y la invocación de los hechos históricos como argumentos en contra del marxismo. Cerdeiras identificaba estos posicionamientos en las formaciones intelectuales que venían propiciando desde la década anterior una articulación entre socialismo y democracia. De alli que utilizara un artículo de Juan Carlos Portantiero publicado en Punto de Vista para ejemplificar este tipo de lecturas. ${ }^{5} \mathrm{Al}$ poner en primer plano los resultados de las experiencias políticas edificadas en nombre de la teoría marxista, Portantiero reemplazaba los requerimientos de la destrucción del marxismo con una mera comprobación empírica. En contraposición, el trabajo que se proponía la revista era el de un pensamiento sin concesiones sobre el agotamiento de las capacidades políticas del marxismo. Se trataba de un modo de procesar el problema de la crisis del marxismo que si, por un lado, entrañaba mayor potencial para un recomienzo de la tradición, por el otro

5. Juan Carlos Portantiero, "Socialismo y democracia. Una relación dificil", en Punto de Vista, n²0, 1984, pp. 1-5. 
expresaba cierto grado de autodestrucción: "el convencimiento de que el marxismo ha sido la última gran creación de la politica, nos obliga a ubicarnos en un lugar que tiene la incomodidad propia de aquel que está destruyendo su propio punto de apoyo". ${ }^{6}$

Otros artículos escritos por Cerdeiras para los primeros números de la revista nos dejan ver que esta destrucción del marxismo tenía como una de sus consecuencias fundamentales el replanteo de los principios de la política. Deudora del trabajo desarrollado por Badiou en la segunda mitad de la década de 1980, dicha revisión apuntaba fundamentalmente al reemplazo de los principios que había sostenido el marxismo-leninismo en el siglo XX por otros que mantuvieran el horizonte de una politica emancipatoria. Cerdeiras apuntaba principalmente a la concepción según la cual la intervención política descansa sobre una correspondencia entre fines y medios. Es decir, lo que se debía dejar atrás era el esquema militante que hacía del programa y la línea de acción elementos indispensables para alcanzar objetivos programáticos. Frente a ello, proponía una concepción de la política como invención y creación de lo radicalmente nuevo. Se trataba de una idea de la política como posibilidad, según la cual "toda vez que el lazo social consistente se conforma suturando (abrochando, cerrando) una inconsistencia radical, la política debe ubicarse precisamente en el lugar donde se des-une el lazo, provocándose la liberación de los acontecimientos imprevisibles". ${ }^{7}$ Estos artículos resultan de interés ya que permiten constatar los efectos que este replanteo tenía en los modos de aproximarse a las luchas sociales y politicas de la sociedad argentina. En algunos casos, se trataba de una revisión de formas tradicionales de lucha. Este era el caso de las protestas de los trabajadores despedidos por las empresas privatizadas. Según Cerdeiras, los modos de despliegue de estas protestas (ocupación de la calle, movilización a instituciones de gobierno) y los formas de encuadrar dicho despliegue (condena al capitalismo, demanda de justicia social) no representaban ningún suplemento al estado de cosas existente. Lo que se requería, por tanto, era una acción política que contrarrestara los intentos de hegemonización dando lugar a nuevas fuerzas y trayectorias que pudieran lentamente ir tomando cuerpo en el entramado social. En otros casos, se trataba de jerarquizar luchas que sí parecian proporcionar un suplemento a la situación. Este era el caso de las "marchas del silencio" por la muerte de María Soledad Morales, en las que se verificaba una operación positiva de despolitización (la

6. Raúl Cerdeiras, "Hacia un pensamiento político de la política", en Acontecimiento, $\mathrm{n}^{\circ} 1,1991$, p. 22.

7. Raúl Cerdeiras, "Hacer nuevas formas de política", en Acontecimiento, n² 2, 1991, p. 22 . 
manifestación que no soporta los discursos que intentan contenerla o significarla), y el de las Madres de Plaza de Mayo, cuya constitución (inorgánica) y su demanda ("aparición con vida") desbordaban los marcos de lo decible y representable.

Si los artículos de Cerdeiras intentaban procesar los efectos de la crisis del marxismo dentro de la cultura de izquierdas, otro conjunto de textos publicados en los primeros números de la revista enfrentaron las consecuencias del derrumbe del socialismo real en las narrativas políticas que se volvieron hegemónicas en la primera mitad de la década de 1990. Una de esas narrativas era la del triunfo de la democracia sobre los regímenes totalitarios y la representación como la forma necesaria de articulación política. Al respecto, Acontecimiento reprodujo un artículo sobre la tesis del fin de la historia de Francis Fukuyama escrito por los jóvenes historiadores de la UBA Roy Hora, Ignacio Lewkowicz, Julio Vezub y Fabio Wasserman. ${ }^{8}$ Con la inclusión de este texto, la revista se diferenciaba, por supuesto, de la hipótesis celebratoria de Fukuyama. No era menos crítica, sin embargo, con las respuestas que dicha hipótesis solía cosechar en el campo de la izquierda intelectual. Según este grupo de historiadores, el hecho de que la izquierda opusiera la continuidad de la historia a su finalización daba cuenta de que el marxismo aún seguía atado a una filosofía de la historia. En sintonía con lo sostenido por Cerdeiras, los historiadores apostaban por la relocalización del marxismo en un espacio que pudiera abrirse "a la dimensión de las hipótesis, de la decisión sin garantías preestablecidas, de una libertad cuyo régimen jamás fue soñado". ${ }^{9}$ En un sentido similar, un artículo relevante de Cerletti apuntaba contra el diagnóstico epocal de la crisis de representación. ${ }^{10}$ La idea de Badiou de que la representación "anula las diferencias y sutura las contradicciones de lo social produciendo la unificación imprescindible (orden de la sociedad civil) para el funcionamiento del todo político (orden del Estado)"11 permitía caracterizar como no politica la demanda de mayor control ciudadano de los dirigentes políticos y mayor transparencia en la gestión del Estado. Si la lógica representacional esterilizaba experiencias potencialmente disruptivas, la militancia política no debía estar orientada a la recomposición de un sistema aparentemente en crisis. A contramano del sentido común de la época, Cerletti intentaba despegar la concepción de la política como

8. Roy Hora, Ignacio Lewkowicz, Julio Vezub y Fabio Wasserman, “¿Fin de la historia? La politica no se rinde”, en Acontecimiento, $\mathrm{n}^{\circ} 1,1991$, pp. 47-59.

9. Ibid., p. 59.

10. Alejandro Cerletti, "La crisis de la representación política", en Acontecimiento, $\mathrm{n}^{\circ}$ 5, 1993, pp. 66-70.

11. Ibid., p. 68. 
la gestión realista y el reaseguro del lazo social para orientarla hacia la idea de la creación y el corte. La otra narrativa enfrentada en los primeros números de la revista, solidaria de la de la democracia y la representación, era la del retorno de la ética y la primacía de los derechos humanos. En este caso, no se trató de artículos escritos por miembros o colaboradores de la revista, sino de traducciones realizadas por Cerdeiras de textos de los referentes franceses de Acontecimiento. En el número 2 fue publicado un fragmento de Utopie et liberté: les droits de l'homme: une idéologie? de Benasayag y el número 8 estuvo dedicado integramente a la reproducción de L'éthique. Essai sur la conscience du mal de Badiou. ${ }^{12}$ En su contexto de origen, los dos textos habian representado intervenciones críticas frente a la consolidación del discurso ético y humanitario resultante del derrumbe del socialismo real y expansión global del capitalismo. Si bien ambos textos eran contundentes en torno al mencionado proceso, el de Badiou se destacaba tanto por su tenor como por su agudeza. Tal como afirma en el prefacio a la edición inglesa del texto, L'éthique había sido concebido como un "panfleto" frente a lo que identificaba como una "contrarrevolución intelectual" basada en el "terrorismo moral" (Badiou, 2001, pp. liii-liv). Con estas traducciones, Acontecimiento difundia intervenciones fuertes que caracterizaban negativamente la suposición de un ser humano general, la subordinación de la política a la ética y la definición del bien a partir del mal, y que -a contrario sensu- proponían la identificación del hombre a partir de sus verdades singulares, la definición del mal a partir del bien y la ubicación de la ética en situaciones concretas.

Entre las reflexiones de Cerdeiras sobre los efectos del derrumbe del socialismo real en el campo de las izquierdas y las intervenciones de miembros y colaboradores de la revista en torno a las narrativas políticas hegemónicas, es posible recortar una serie de textos en los cuales se procesaban los problemas específicos de la práctica política. En un sentido consecuente con el resto de las intervenciones propiciadas por la revista, los materiales relativos a esta problemática se caracterizaron por la jerarquización del compromiso militante y la delimitación de una práctica política a distancia del Estado y la forma-partido. Algunas de estas aproximaciones se desplegaron en un plano eminentemente teórico. Así como en el primer número el grupo de historiadores de la UBA, a la pregunta sobre el fin de la historia, le oponian el dictum la política no se rinde, en el número siguiente Lewkowicz sostendrá que

12. Miguel Benasayag, "Utopía y libertad. Los derechos del hombre: ¿una ideología?", en Acontecimiento, ${ }^{\circ}$ 2, 1991, pp. 14-22 y Alain Badiou, La ética. Ensayo sobre la conciencia del mal (texto completo), en Acontecimiento, $n^{\circ} 8,1994$, número especial. 
la militancia no se rinde ante la caducidad de la política partidaria. ${ }^{13}$ Asumiendo el agotamiento de la experiencia leninista pero sosteniendo el carácter axiomático de la militancia, Lewkowicz le otorgaba a la militancia la tarea de "escribir ahí, en el vacío específico de la situación, un enunciado supernumerario capaz de suplementar (es decir, trastornar) la estructura de la situación". ${ }^{14}$ Otras aproximaciones al problema de la práctica política consistieron en el seguimiento de algunas experiencias concretas. Nos hemos referido anteriormente al lugar ocupado en los textos de Cerdeiras por las Madres de Plaza de Mayo y las "marchas del silencio". Además de estas dos experiencias, la revista le otorgó un espacio significativo a modos de intervención en los que se percibía un fuerte compromiso militante y una distancia del Estado y del partido. Una de ellas era el trabajo realizado en la Cárcel de Devoto, con el cual -como vimos- Zerba estaba fuertemente comprometido. Al respecto, Acontecimiento publicó entrevistas a Guillermo Costa Vidán y Sergio Schoklender, dos presos que participaban activamente del CUD y del Centro de Información Aplicada (CINAP), ${ }^{15}$ así como un artículo en el cual Zerba recuperaba estas experiencias en términos de un productivo alejamiento del repertorio militante tradicional de la izquierda. ${ }^{16}$ Por otra parte, la revista acompañó el trabajo realizado por la Asociación Gays por los Derechos Civiles, la cual tenía por aquellos años una fuerte presencia en el ámbito de la militancia y en el debate público por impulsar acciones contra la discriminación a la comunidad homosexual. Sobre esta experiencia, se publicó una entrevista al referente de la Asociación Carlos Jáuregui en la que las preguntas estaban orientadas principalmente hacia la relación de este movimiento con el marxismo, el Estado y los partidos. ${ }^{17}$

$$
* * *
$$

III. El balance realizado por Cerdeiras en 1994 en ocasión de los tres años de Acontecimiento constituye un material privilegiado para dar

13. Ignacio Lewkowicz, “¿Fin del partido? La militancia no se rinde”, en Acontecimiento, $\mathrm{n}^{\circ}$ 2, 1991, pp. 32-46.

14. Ibid., p. 44.

15. Guillermo Costa Vidán, "Reflexiones sobre el CUD y el CINAP", en Acontecimiento, $\mathrm{n}^{\circ}$ 5, 1993, pp. 72-74 y "Entrevista a Sergio Schoklender", en Acontecimiento, ${ }^{\circ}$ 9, 1995, pp. 58-67.

16. Diego Zerba, "Experiencias de autogestión en cárceles", en Acontecimiento, n 5 , 1993, pp. 75-79.

17. "La irrupción política de los movimientos gays. Entrevista a Carlos Jáuregui", en Acontecimiento, $\mathrm{n}^{\circ}$ 7, 1994, pp. 34-47. 
cuenta del pasaje a una nueva fase de la revista. ${ }^{18}$ En primer lugar, en tanto análisis retrospectivo, este balance nos permite volver a algunos de los impulsos que condujeron a la publicación de la revista y algunas características que marcaron sus primeros años. Resulta interesante el modo en el que Cerdeiras vinculaba el tipo de trabajo que se imponía dentro de la cultura de izquierda con la materialidad de la revista. A una empresa que tenía como fin el fomento del pensamiento activo y la subversión de los principios de la política, le correspondía una forma de intervención austera y despojada:

Quise fabricar un "ladrillo" poblado de letras y textos largos, para desarrollos pensados, en detrimento del protagonismo de la imagen y la lectura rápida y fácil, esa lectura de empalme directo con los preconceptos ya establecidos. ${ }^{19}$

Lo mismo cabe decir de las formas en las cuales Cerdeiras delimitaba el espacio de la revista en el marco de las tradiciones de la izquierda argentina. Mencionamos en la sección anterior las particularidades de la posición que pregonaba la destrucción del marxismo sin plegarse a la gramática política de la democracia y la ética. Al ser reconstruida años después, la separación de la intelectualidad progresista se volvía aún más categórica:

nos ha costado sangre, sudor y angustia tener que dar por clausurada esa experiencia [la marxista], y al mismo tiempo no ceder al aluvión de intelectuales (ex marxistas) que se pasaron al campo de sus enemigos de ayer, descubriendo los "verdaderos" valores de su ideología que no pudieron apreciar en su momento. ${ }^{20}$

El sostenimiento de esta caracterización puede ser comprendida en el marco de la conformación de un polo opositor al menemismo de perfil progresista. Nos referimos al Frente Grande, que habia ganado recientemente las elecciones constituyentes en la Capital Federal convirtiéndose en la tercera fuerza política a nivel nacional. Al igual que había ocurrido a comienzos de la década de 1990 con los intelectuales cercanos al alfonsinismo, Cerdeiras veía en el Frente Grande una experiencia que renunciaba de antemano a la emancipación al sostener una política basada en la representación, el partido y el Estado. Mientras la supuesta novedad

18. Raúl Cerdeiras, "Tres años de Acontecimiento", en Acontecimiento, n 7, 1994, pp. 22-34.

19. Ibid., p. 22.

20. Ibid., p. 23. 
en la política argentina no era más que una expresión aggiornada de la vieja política, el balance de Cerdeiras mencionaba un acontecimiento producido simultáneamente a la escritura del texto: la irrupción en el sudeste mexicano de una experiencia que no podía ser contenida por los principios y discursos de la política tradicional. El hecho de que el Ejército Zapatista de Liberación Nacional (EZLN) renunciara a la toma del poder, y fuera por ello denostada por las formaciones clásicas de la izquierda, colocaba a la experiencia zapatista en un primer plano de la atención de la revista.

Efectivamente, las intervenciones desarrolladas por Cerdeiras en la segunda mitad de la década de 1990 estuvieron dominadas por las vicisitudes de la experiencia del EZLN. En primer lugar, lo ocurrido en Chiapas fue rápidamente caracterizado como la expresión de un nuevo pensamiento de la política. La afinidad entre los postulados que la revista venía difundiendo y una parte significativa del discurso y la praxis del EZLN redundó en la tesis de que "Chiapas marca el advenimiento del primer pensamiento de la política que se hace cargo del agotamiento de las viejas formas de la política y promueve un vigoroso esfuerzo por reinventar la política de emancipación". ${ }^{21}$ Cerdeiras orientaba la interpretación de la experiencia zapatista en el sentido de un pensamiento en interioridad de la política, una fidelidad a la idea de emancipación, el sostenimiento de una decisión subjetiva y la renuncia a la representación. Será precisamente sobre este último aspecto que se concentrarán mayormente los análisis realizados por Cerdeiras durante estos años. La insistencia de la revista en este tópico desde sus orígenes, así como la constitución en Argentina de una fuerza progresista que no hacía sino reforzar la representación, transformó al discurso del EZLN en un elemento que permitía combatir la idea de que la política transformadora debía canalizarse necesariamente a través del Estado, los partidos y la gestión. Lo que había irrumpido en Chiapas no era la tradicional negociación entre víctimas y el Estado a través de sujetos que actuaban como representantes de las primeras ante el segundo, sino que se había desplegado una política en la que cifraban la fidelidad a una causa y el compromiso axiomático de la militancia. A diferencia de proyectos como el del Frente Grande, que hacía de los resultados de la gestión el elemento dinamizador de la transformación social, la experiencia del EZLN demostraba que podian obtenerse efectos sociales concretos a través del primado de la causa sobre la negociación.

Además de la del EZLN, otra experiencia política acogida por la revista fue la del movimiento 5Ø1. De cara a las elecciones presidenciales de 1999, un grupo de jóvenes militantes de la UBA que venían discutiendo

21. Raúl Cerdeiras, "La política. Chiapas", en Acontecimiento, n 9, 1995, p. 44. 
sobre prácticas políticas más allá del voto, deciden viajar a más de 500 kilómetros a los fines de estar exceptuados de la obligación de emitir el sufragio. ${ }^{22}$ A diferencia de la del EZLN, con la cual la revista no tenía relaciones concretas, el vínculo de Acontecimiento con el movimiento $5 \varnothing 1$ era estrecho. Varios de los estudiantes que formaban parte del movimiento participaban a su vez en los grupos de estudios coordinados por Lewkowicz. Las ideas de Lewkowicz, y por ende varias de las sostenidas por la revista como premisas de su intervención, son fácilmente detectables en el documento con el que el movimiento $5 \varnothing 1$ se presentó públicamente y convocó a la acción a realizarse el día de la elección presidencial. El texto en cuestión, titulado "Carta a los no votantes", fue reproducido en el número 18 de la revista. ${ }^{23}$ Bajo la hipótesis de que "la politica tiene que ver con la transformación y no con la mera gestión de lo existente", ${ }^{24}$ el documento llamaba a dar un paso más en la impugnación de la democracia representativa y poner en movimiento un dispositivo de discusión colectiva sobre un nuevo pensamiento de la política. La discusión que intentaba propiciar el documento estaba centrada fundamentalmente en la disputa alrededor del significado de la democracia. Esto es, que la verdadera democracia es aquella en la que todos los miembros de la comunidad debaten y participan en las decisiones que les atañe en tanto sujetos, y no las formas actuales en las que las perspectivas de cambio real y efectivo están excluidas. Consideraciones de este tipo no podian resultar sino disruptivas en el debate político de la Argentina de la posdictadura, que giraba en torno al binomio democracia-dictadura y que consideraba irresponsable cualquier cuestionamiento al sistema político consolidado a partir de 1983. Es por ello que el documento del $5 \varnothing 1$ dedicaba un espacio significativo a evidenciar el carácter ficcional de las formas democráticas contemporáneas y desplazar la contradicción hacia "el dilema actual: pobre democracia vs. nueva sociedad a inventar". ${ }^{25}$

Finalmente, en esta segunda etapa de la revista se volverá sistemática aquella aproximación a las Madres de Plaza de Mayo como una experiencia en la que se constataban los nuevos principios de la política. En el número 13 de la revista, Cerdeiras publicó sus "20 tesis acerca de Madres de Plaza de Mayo". ${ }^{26}$ Alli, la caracterización de dicha

22. Para una reconstrucción de la experiencia del 5Ø1, ver Bergel (2000). Para un análisis del $5 \varnothing 1$ en el marco de las corrientes autonomistas argentinas, ver Bergel (2009).

23. 5Ø1. "Carta a los no votantes", en Acontecimiento, n 18, 1999, pp. 56-61.

24. Ibid., p. 56.

25. Ibid., p. 59.

26. Raúl Cerdeiras, “20 tesis sobre Madres de Plaza de Mayo y algo más”, en Acontecimiento, $\mathrm{n}^{\circ} 13,1997$, pp. 65-67. 
experiencia en el marco del agotamiento de la política comunista era categórica: "Madres de Plaza de Mayo es el acontecimiento político más importante de los últimos veinte años en nuestro país". ${ }^{27}$ La conceptualización de las Madres de Plaza de Mayo como acontecimiento político cobraba sentido en su ubicación en el trayecto que iba del fin del ciclo marxista-leninista a nuevas formas de pensar y hacer la política. Al haberse puesto a distancia del Estado, las Madres de Plaza de Mayo habían impedido el funcionamiento de los tres niveles propios de la política moderna: que el objetivo de la política es el acceso al poder del Estado, que la política se realiza a través de los partidos y que las relaciones entre pueblo y Estado están mediados por la representación. Era precisamente esa distancia la que volvía a su discurso y su acción difíciles de encuadrar en los modos tradicionales de la política. En suma, la experiencia de Madres de Plaza de Mayo daba cuenta de que "la política adviene cuando se corta el trípode Estado-representación-partidos". ${ }^{28}$ Ahora bien, la valoración de las Madres de Plaza de Mayo irá en paralelo a la crítica a la imposición de un discurso sobre la politica revolucionaria centrado en la memoria. Como es sabido, a mediados de la década de 1990 se produjo una transformación en las representaciones sobre la década de 1970 que se habían codificado en la transición a la democracia. En el marco de las fuertes desigualdades generadas por las políticas neoliberales, el surgimiento de la agrupación HIJOS y la proliferación de artefactos culturales que recuperaban la voz de los militantes propiciaron una politización de la figura del desaparecido. Si bien algunos de los aspectos del nuevo relato sobre la década de 1970 podían tener puntos de contacto con el tipo de intervención de la revista, Cerdeiras desplegará una mirada fundamentalmente crítica sobre el fenómeno. En un artículo sugerentemente titulado "Contra la memoria", ${ }^{29}$ se establecía una relación de tipo negativa entre el deber de recordar el horror y las política activas y emancipatorias. Apuntalado por la hipótesis de Badiou de que "la memoria es una categoría propia de las políticas reaccionarias", ${ }^{30}$ Cerdeiras intentaba demostrar que la consigna del "Nunca más" y la idea del horror como impensable impedian un pensamiento politico sobre el terrorismo de Estado. En términos más específicos, Cerdeiras también enfatizaba la dimensión puramente fáctica y jurídica de la verdad y la justicia perseguida por el movimiento de derechos humanos y gran parte de la sociedad argentina. Contra este dispositivo, Cerdeiras establecía un horizonte en el que el

27. Ibid., p. 65.

28. Ibid., p. 66.

29. Raúl Cerdeiras, “Contra la memoria”, en Acontecimiento, n 10, 1995, pp. 30-34. 30. Ibid., p. 30. 
problema del terrorismo sea procesado en términos políticos (el terror como inseparable de la política de Estado) y en el que la memoria sea reemplazada por la fidelidad (la política revolucionaria como gesto de ruptura que inaugura lo nuevo).

En los últimos años de la década de 1990, todas estas experiencias que daban cuenta del agotamiento de la política marxista-leninista y de la posibilidad de un nuevo modo de intervención fueron abordadas en conjunto como parte de una misma secuencia politica. Volviendo a las tesis sobre las Madres de Plaza de Mayo, Cerdeiras vinculaba esta experiencia con la de los cortes de ruta que comenzaban a desarrollarse en distintos lugares del país. Por un lado, ambas representaban formas de lucha que resultaban dificiles de encuadrar en los viejos patrones de la política. Al igual que las Madres, los piqueteros o fogoneros actuaban fuera de los partidos y los sindicatos, no se planteaban como objetivo el acceso a cargos en el Estado y obligaban a las autoridades a acercarse al espacio de la irrupción política. Por el otro, se verificaba en todas estas experiencias el mismo esfuerzo de los medios, los partidos y las instituciones por revertir el proceso de conversión de las víctimas en sujetos libres. De allí que todas las personas que formaban parte de estas experiencias fueran objeto de un proceso de victimización que sentaba las bases para la captura estatal. Concluía Cerdeiras: "Madres de Plaza de Mayo, Chiapas, cortadores de viejas rutas, se deben tejer alrededor de la idea de que son irrupciones, confusas, quizás aún, de pensamiento y experiencias que van delineando una nueva forma de pensar-hacer la política". ${ }^{31}$ Lo mismo ocurría con la caracterización de todas estas experiencias en el marco del regreso de la ética y los derechos del hombre. En otro artículo que versaba sobre la temática, Cerletti profundizó en aquel argumento que, más allá de la impugnación de la narrativa ética, insistía en la necesidad de éticas singulares y situadas. Si en lugar de ideología legitimadora del capitalismo, la ética era concebida como la fidelidad a un acontecimiento, podía afirmarse que "las Madres de Plaza de Mayo o el EZLN, en Chiapas, marcan un trayecto ético". ${ }^{32}$ Es decir, que en ambos se podía constatar el sostenimiento de un pensamiento y una acción que eran consecuentes con una ruptura en el estado de dominación y que no podían ser interpretadas a través de la lógica de la gestión y la representación.

$$
* * *
$$

31. Raúl Cerdeiras, "20 tesis sobre Madres de Plaza de Mayo y algo más”, en Acontecimiento, $\mathrm{n}^{\circ} 13,1997$, p. 67.

32. Alejandro Cerletti, "Política, gestión y ética", en Acontecimiento, n 12, 1996, p. 34. 
IV. Así como el levantamiento zapatista había propiciado una inflexión en los modos en los que Acontecimiento había analizado distintas expresiones politicas en la primera mitad de la década de 1990, la revuelta popular de diciembre de 2001 llevó a un nuevo plano interpretativo la secuencia política desplegada en la segunda mitad de la década anterior. En un importante artículo que da cuenta de este movimiento, Cerdeiras caracterizaba retrospectivamente a estas experiencias como elementos que habian formado parte de un lento debilitamiento de la impotencia política característica de la década de $1990 .{ }^{33}$ La revuelta de diciembre de 2001 había generado un espacio a partir del cual ese trayecto anterior podía ordenarse y abrirse a una nueva radicalidad de la política. Por eso podía afirmarse que "el 19/20 de diciembre se inscribe en una línea que articula una serie de acontecimientos que, comenzando por las Madres de Plaza de Mayo, pasa por el movimiento piquetero y el colectivo 5Ø1". ${ }^{34}$ La confluencia entre el ciclo político abierto en diciembre de 2001 y el programa político sostenido por Acontecimiento conducirá a una reorientación de los problemas atendidos por Cerdeiras. Una vez iniciada una política radical autónoma, la mirada no estará tan puesta en las formas gestionarias y representativas de la política-impugnadas por el propio movimiento- como en la disputa acerca de la interpretación e intervención sobre dicha radicalidad. En este sentido, Cerdeiras establecía una posición diferenciada tanto de la izquierda tradicional, que hacía de la política un medio para la toma del poder del Estado, como de nuevas formaciones autonomistas, que disolvía a la política en la multiplicidad de lo social. Frente a las organizaciones trotskistas y el autonomismo inspirado en la obra de Negri, Cerdeiras apostaba a un pensamiento de la política como "un pensar-hacer con capacidad de subvertirse a sí misma y de esta manera interrumpir la vigencia de los lazos sociales existentes" ${ }^{35}$ Los términos de esta disputa se replicaban en las reflexiones a propósito de los modos de vincularse con la experiencia de las asambleas barriales. Según Cerdeiras, se trataba de un espacio privilegiado de intervención en tanto allí se jugaba en gran medida el porvenir del ciclo político abierto en diciembre de 2001:

Las asambleas barriales son el efecto político más importante del "cacerolazo", es algo así como el moño con el que se cierra esta secuencia de la nueva política que se abre con el acontecimiento "Madres de Plaza de Mayo". ${ }^{36}$

33. Raúl Cerdeiras, "La política que viene", en Acontecimiento, $\mathrm{n}^{\circ}$ 23, 2002, pp. 5-48.

34. Ibid., p. 37.

35. Ibid., p. 28.

36. Ibid., p. 45. 
En tanto punto de partida de la política por venir, la asamblea no debía ser capturada por la lógica de la vieja izquierda, que intentaba captar militantes para sus organizaciones, ni por la de los nuevos autonomismos, que veían alli la irrupción de una potencia capaz de resquebrajar la dominación biopolítica del capital. ${ }^{37}$

La recomposición del sistema político propiciado por el kirchnerismo y el desarrollo de un ciclo político de corte progresista en América Latina marcaron el inicio de la larga última etapa de la revista. En los números que siguieron a la revuelta de 2001, Cerdeiras y colaboradores advertirán, primero, sobre la disipación de las fuerzas que se habian desplegado en diciembre de aquel año, ${ }^{38}$ y finalmente, sobre el agotamiento de la experiencia asamblearia. ${ }^{39} \mathrm{Al}$ igual que otras expresiones de la izquierda intelectual argentina, Acontecimiento analizará al kirchnerismo y al ciclo político progresista latinoamericano en clave de recomposición de las relaciones capitalistas y restauración de las formas de la política tradicional. Sin embargo, la mediación badiouana en los análisis de Acontecimiento perfilará una intervención centrada en el carácter reactivo del nuevo ciclo político, ${ }^{40}$ la subordinación de la política al Estado y los partidos, ${ }^{41}$ la borradura del acontecimiento $2001^{42}$ y la suplantación de una verdadera igualdad por un discurso centrado en la equidad y los derechos. ${ }^{43}$ Los textos centrales de Cerdeiras y las declaraciones publicadas de cara a las elecciones desarrolladas en Argentina dan cuenta tanto de la apuesta por una política radical autónoma como de cierta autoconciencia acerca de la marginalidad de un programa como el de la revista en el nuevo contexto político argentino y latinoamericano. ${ }^{44}$

37. En los números siguientes, Cerdeiras seguirá polemizando con la obra de Negri y sus recepciones locales, ver "La ontología biopolitica de Imperio", en Acontecimiento, $\mathrm{n}^{\circ}$ 23-24, 2003, pp. 54-74.

38. "La situación política. Declaración del Grupo Acontecimiento", en Acontecimiento, n²6, 2003, pp. 19-23.

39. Andrés Pezzola, "Derrota, fracaso o agotamiento de la experiencia asamblearia", en Acontecimiento, $n^{\circ}$ 27, 2004, pp. 31-41.

40. Raúl Cerdeiras, "La política de emancipación frente a los nuevos gobiernos latinoamericanos", en Acontecimiento, $\mathrm{n}^{\circ} 32,2006,44-51$.

41. Raúl Cerdeiras, "La contracumbre o la impotencia de la vieja política", Acontecimiento, $\mathrm{n}^{\circ} 31,2006,23-36$.

42. Víctor Militello, "Carta abierta: la adjunción de un sujeto al gobierno", en Acontecimiento, $\mathrm{n}^{\circ}$ 36-37, 2009, 75-90.

43. Alejandro Cerletti, "Igualdad y equidad en las políticas sociales y educativas", en Acontecimiento, $\mathrm{n}^{\circ}$ 38-39, 2010, 95-104.

44. Raúl Cerdeiras, "Cómo piensa la política el Grupo Acontecimiento", en Acontecimiento, $\mathrm{n}^{\circ}$ 29-30, 2005, 7-42; Grupo Acontecimiento. "La afirmación de otra política 
Durante estos años, Acontecimiento seguirá acompañando la trayectoria del EZLN -dando lugar en sus páginas a la Otra Campaña y la Sexta Declaración de la Selva Lacandona- ${ }^{45}$ y acogerá nuevas experiencias como la del Proyecto Nuestramérica-Movimiento 13 de Abril de Venezuela -una organización de corte autonomista liderada por el ex-ministro Roland Denis- ${ }^{46}$, el Movimiento 15-M en España ${ }^{47}$ y el ciclo de revueltas conocido como "primavera árabe". ${ }^{48}$ Además de las hipótesis que delinearon la intervención de la revista en su última etapa, la obra de Badiou estará presente de dos maneras en este nuevo contexto politico. Por un lado, Cerdeiras la opondrá diametralmente a las ideas de Ernesto Laclau, la cual se convertiría en aquellos años en un insumo teórico significativo en los debates intelectuales alrededor del kirchnerismo. En el año 2003, la revista publicó un debate entre Cerdeiras y Laclau que había tenido lugar en la UBA. Alli, Cerdeiras apelaba a Badiou para afirmar que la obra de Laclau impedía pensar la novedad política debido al lugar preponderante que tenía en ella la cuestión estructural. A modo de respuesta, Laclau le señalaba a Cerdeiras la dificultad de un pensamiento sobre la política que -al modo de Badiou- le otorgaba un carácter cerrado a la totalidad anterior al advenimiento. ${ }^{49}$ Por el otro, la revista se convertirá en un espacio de disputa con otras lecturas de Badiou realizadas en Argentina. En el mismo contexto en el cual Acontecimiento utilizaba la obra de Badiou para desestimar la aparente radicalidad del kirchnerismo, lecturas novedosas de dicha obra propiciaban una apertura a las nuevas experiencias políticas en Argentina y América Latina. En sus últimos números, la revista apuntará fundamentalmente contra el trabajo de Roque Farrán, intentando demostrar que la irrupción de los gobiernos progresistas resultaba totalmente ajena a la idea de acontecimiento en la obra de Badiou. ${ }^{50}$

de emancipación", en Acontecimiento, n 36-37, 2009, 7-29; Cerdeiras, Raúl. "Acontecimiento cumple 20 años de existencia", en Acontecimiento, $\mathrm{n}^{\circ} 40,2011,7-20$; Grupo Acontecimiento. "Que se vayan todos", en Acontecimiento, n 45, 2014, 9-14.

45. Elsa González y Gabriel D’orio, "Otras campañas. La apuesta zapatista y los avatares de la nueva política”, en Acontecimiento, $\mathrm{n}^{\circ}$ 29-30, 2005, 43-51; Cerdeiras, Raúl. "La autonomía y el pensamiento político emancipativo en la encrucijada zapatista", en Acontecimiento, $n^{\circ} 31,2006,3-22$.

46. "Dossier Venezuela", en Acontecimiento, n 33-34, 2007, 7-102.

47. Martín López, "El hastío de los cualquiera. Sobre España 15-M", en Acontecimiento, $\mathrm{n}^{\circ} 40,2011,39-43$.

48. Martín López, "Lo que falta, lo que viene", en Acontecimiento, n 43-44, 2013, 61-73.

49. "Debate Laclau-Cerdeiras", en Acontecimiento, n 24-25, 2003, 78-100.

50. Martín López, "La posibilidad actual de un Estado emancipador. Acerca de un trabajo de Roque Farrán", en Acontecimiento, n 46, 2015, 53-77. 
A modo de cierre, podemos afirmar que Acontecimiento constituyó una experiencia significativa desarrollada durante veinticinco años en el seno de la izquierda intelectual argentina. Expresión de la recepción argentina de Badiou, la revista analizó la política argentina y regional desde el prisma de las tesis y los conceptos de la obra del filósofo francés. En un primer momento, la posición de Badiou frente al agotamiento de la experiencia del socialismo real le permitió a la revista desarrollar una intervención que escapaba a las dos reacciones habituales de la izquierda frente al derrumbe: la persistencia en los marcos del marxismo-leninismo y el acoplamiento al nuevo orden político y económico. Diferenciada tanto del trotskismo como del socialismo democrático, Acontecimiento apostó a un replanteo de los principios de la política en el sentido de una intervención a distancia de los partidos y el Estado. Como pudimos ver, este ejercicio de replanteo trajo aparejada una caracterización de las luchas politicas llevadas a cabo en la sociedad argentina en la que se señalaban los limites de los repertorios tradicionales y se valoraban nuevas formas de intervención. Asimismo, en un sentido más amplio, la obra de Badiou habilitó un posicionamiento escéptico tanto frente a las bondades de la democracia liberal luego del "siglo de los totalitarismos" como a la necesidad de un retorno a la ética y la los derechos humanos. En un segundo momento, la revista leerá en experiencias como el EZLN, el colectivo $5 \varnothing 1$, las Madres de Plaza de Mayo y el movimiento piquetero los indicios de una nueva secuencia política desplegada a distancia de los partidos, el Estado y la representación. Iniciado el proceso político radical y autónomo en diciembre de 2001, la revista encontrará en la obra de Badiou ya no el sustento para una crítica general a las formas de la político sino una serie de insumos teóricos productivos a los fines de discutir con otras interpretaciones realizadas por las izquierdas argentinas. Al igual que ocurrió en otras expresiones de la izquierda intelectual, el kirchnerismo y el ciclo político progresista latinoamericano redundaron en un debilitamiento, y posterior agotamiento, de la experiencia de Acontecimiento.

\section{Referencias bibliográficas}

Ashton, P. (2006). "A Bibliography of Work on and Alain Badiou in English". Cosmos and History: The Journal of Natural and Social Philosophy, 2 (1-2), 213-226.

Ahston, P., A.J. Bartlett y J. Clemens (2006). Masters \& Disciples: Institution, Philosophy, Praxis. En P. Ahston, A.J. Bartlett y J. Clemens (eds.). The Praxis of Alain Badiou (3-12). 
Badiou, A. (2001). "Preface to the English Edition". En Ethics. An Essay on the Understanding of Evil (liii-1xiii). Verso.

Bartlett, A.J. y J. Clemens (2010). Introduction: Badiou's form. En A.J. Bartlett y J. Clemens (eds.). Alain Badiou: Key Concepts (1-7). Acumen.

Beigel, F. (2003). Las revistas culturales como documentos de la historia latinoamericana. Utopía y Praxis Latinoamericana, 8 (20), 105-115.

Bergel, M. (2000). 5Ø1. Balance de una experiencia politica. El Rodaballo. Revista de politica y cultura, 10, 3-9.

Bergel, M. (2009). En torno al "autonomismo argentino". En C. Albertani, G. Rovira y M. Modonesi (coords.). La autonomía posible. Emancipación y reinvención de la politica (285-325). Universidad Autónoma de México.

Bosteels, B. (2001). "Alain Badiou's Theory of the Subject: Part I. The Recommencement of Dialectical Materialism?". Pli. The Warwick Journal of Philosophy, 12, 200-229.

Bosteels, B. (2011). Badiou and Politics. Duke University Press.

Feltham, O. (2004). Singularity happening in politics: the Aboriginal Tent Embassy, Canberra 1972. Communication and Cognition, 37 (1), 225-245.

Feltham, O. (2010). Biography and early works. En: A.J. Bartlett y J. Clemens (eds.). Alain Badiou: Key Concepts (8-10). Acumen.

Hallward, P. (1998). Generic sovereignty. The philosophy of Alain Badiou. Angelaki: Journal of the Theoretical Humanities, 3 (3), 87-111.

Hallward, P. (2003). Badiou: A Subject to Truth. University of Minnesota Press.

Pita, A. y M. Grillo (2013). Revistas culturales y redes intelectuales: una aproximación metodológica. Temas de Nuestra América, 54, 177-194.

Pluet-Despatin, J. (1992). Une contribution à l'histoire des intellectuels: les revues. Les Cahiers de l'IHTP, 20, 125-136.

Sarlo, B. (1992). Intelectuales y revistas: razones de una práctica. América: Cahiers du CRICCAL, 9-10, 9-16.

Žižek, S. (1999). Psychoanalysis in Post-Marxism: The Case of Alain Badiou. The South Atlantic Quarterly, 97 (2), 235-261.

Žižek, S. (2003). "Hallward's Fidelity to the Badiou Event". En P. Hallward. Badiou: A Subject to Truth (IX-XIII). University of Minnesota Press. 